\title{
A systematic review and meta-analysis of the impact of GLP-1 receptor agonists and SGLT-2 inhibitors on cardiovascular outcomes in biologically healthy older adults
}

\author{
WILLIAM D STRAIN,${ }^{1}$ JONATHAN GRIFFITHS²
}

\begin{abstract}
Background: Unintentional weight loss is a hallmark of frailty and is associated with poor outcomes in older adults with type 2 diabetes. As such, the role of pharmacological therapies that facilitate weight loss - namely, sodiumglucose co-transporter-2 (SGLT-2) inhibitors and glucagonlike peptide-1 (GLP-1) receptor agonists - remains uncertain in fitter older adults. We performed a systematic review and meta-analysis to evaluate these agents on major adverse cardiovascular events (MACE) in older adults eligible for participation in cardiovascular outcome trials.

Methods: A literature search was performed in MEDLINE, EMBASE, CINAHL, Cochrane Central Registry of Controlled Trials (CENTRAL) and CNKI from inception to 29 June 2020. A class-specific meta-analysis was conducted in older adults (>65 years at recruitment) and compared with the similar analysis in younger ( $<65$ years) adults.

Results: Of 761 unique studies identified, nine met the criteria for inclusion, five using GLP-1 receptor agonists and four with SGLT-2 inhibitors. GLP-1 receptor agonists in older adults were associated with a 15.3\% (OR 0.847 (95\% Cl 0.788 to 0.910$)$ ) reduction in MACE events, similar to the $16 \%$ benefit seen in younger adults. The use of SGLT-2 inhibitors reduced MACE in older participants by $16.9 \%$ (OR 0.831 (95\% $\mathrm{Cl} 0.699$ to 0.989$)$ ), numerically superior to the impact in younger patients (OR 0.936 (95\% Cl 0.787 to 1.113$)$.

Conclusions: GLP-1 receptor agonists and SGLT-2 inhibitors reduced MACE outcomes in older adults who were eligible
\end{abstract}

\footnotetext{
Clinical Senior Lecturer, Institute of Biomedical Science, University of Exeter Medical School; Clinical Lead of Diabetes and Vascular Research Centre; Head of Academic Department for Healthy Ageing, Royal Devon \& Exeter NHS Foundation Trust, Diabetes and Vascular Research Centre, University of Exeter Medical School, Exeter, Devon, UK

Academic Department of Healthcare for Older People, Royal Devon \& Exeter Hospital, Exeter, Devon, UK
}

Address for correspondence: Dr William D Strain Diabetes and Vascular Research Centre, University of Exeter Medical School, Exeter, Devon, EX2 5AX, UK

E-mail: d.strain@exeter.ac.uk

https://doi.org/10.15277/bjd.2021.292 to participate in clinical trials. Whereas this is reassuring for the biologically robust, it should not be extrapolated to frail older adults without further investigation.

Br J Diabetes 2021;21:30-35

Key words: type 2 diabetes, older adults, major adverse cardiovascular events, cardiovascular outcome trials, GLP-1 receptor agonists, SGLT-2 inhibitors

\section{Introduction}

The International Diabetes Federation estimated the number of people living with diabetes worldwide at 463 million in 2019, $85 \%$ of whom had type 2 diabetes. ${ }^{1}$ This figure, however, does not include adults over the age of 80 . In the UK, approximately $20 \%$ of the population over the age of 85 have diabetes and $27 \%$ of people in care homes, accounting for $15 \%$ of the British population living with diabetes. ${ }^{2}$

Frailty - that is, a reduction in physiological reserve with associated sarcopenia, weight loss and functional decline - is a recognised complication of diabetes. Older adults with frailty and diabetes are at a greater risk from medication side effects, polypharmacy and complications due to their co-morbidities. ${ }^{2}$ There has been a recent focus on addressing the over-treatment of frail older adults in order to reduce this risk after the publication of a national stakeholders document for the assessment and target setting, ${ }^{3}$ followed by the adoption of this standard by the National Institute for Health and Care Excellence (NICE; NG158 and NG160) and the General Medical Services Quality Outcome Framework (GMS QOF) for primary care. Within these guidance documents, the use of drugs that promote weight loss are discouraged as a potential risk for progression of frailty. Specifically, the use glucagon-like peptide-1 (GLP-1) receptor agonists and sodium-glucose co-transporter-2 (SGLT-2) inhibitors are highlighted as agents that may carry risk for older adults. It is important to remember, however, that the majority of older adults are not frail. This was recognised in the stakeholders guidance document, which recommended that biologically fit older adults living with diabetes should be treated in a similar manner to their chronologically younger counterparts. ${ }^{3}$ Notwithstanding this, there remain concerns among some healthcare workers that agents facilitating weight loss may augment progression to frailty. 
GLP-1 analogues mimic the incretin response to food, increasing insulin secretion, slowing gastric emptying and promoting satiety. ${ }^{4}$ In the younger population ( $<65$ years of age) these are associated with approximately $4-6 \mathrm{~kg}$ weight loss. ${ }^{5}$ SGLT-2 inhibitors reduce glucose reabsorption in the proximal tubule of the nephron, thereby promoting calorie loss and producing a similar 4-6 kg weight loss. ${ }^{6}$ These medications have been proven to reduce the incidence of major adverse cardiac events (MACE; cardiovascular death, non-fatal myocardial infarction and nonfatal stroke) in high-risk trial populations with type 2 diabetes. ${ }^{7}$

We planned to address the uncertainty as to whether these agents have similar cardiovascular benefits in older patients as is accepted in younger populations, or whether the impact on weight loss promotes frailty thereby failing to show benefit or even being associated with harm. We performed a systematic review and meta-analysis of cardiovascular outcome trials of GLP-1 receptor agonists and SGLT-2 inhibitors, where a priori or post hoc analyses were presented stratified by age.

\section{Methods}

The protocol is registered on the PROSPERO database (CRD42020200601) and is reported in accordance with the reporting guidance provided in the Preferred Reporting Items for Systematic Reviews and Meta-Analyses Protocols (PRISMA-P) statement. 8,9

\section{Eligibility criteria}

Studies were selected according to the following criteria:

- Study design: Only prospective randomised controlled trials were included. Retrospective comparative cohort studies, case-control or nested case-control studies and case series were excluded.

- Participants: Any study that included people with type 2 diabetes and presented an a priori or post hoc age-stratified analysis with an event rate reported for 'older adults'. Any age $\geq 65$ years was regarded as satisfactory for 'older'.

- Interventions: Studies that used a GLP-1 receptor antagonist or SGLT-2 inhibitor as the agent of interest.

- Comparators: The comparator could be placebo or standard care management aiming for glycaemic equipoise.

- Outcome measures: Adjudicated MACE outcome was required. This could be a 3-point MACE (cardiovascular death, non-fatal myocardial infarction or non-fatal stroke), a 4-point MACE (3-point plus hospitalisation for acute coronary syndrome) or MACE+ (3- or 4-point MACE and hospitalisation for heart failure).

\section{Information sources and search strategy}

We searched the following electronic databases: MEDLINE, EMBASE, CINAHL, Cochrane Central Registry of Controlled Trials (CENTRAL) and CNKI from inception to 29 June 2020. Only English language manuscripts were included. The reference lists of included studies were also searched for additional studies.

Screening, selection and data extraction procedure Both authors screened all articles identified from the search independently, starting with titles and abstracts and then full texts were examined in detail. Any disagreements would have been resolved by discussion; however, both authors reached consensus on first review.

\section{Risk of bias assessment}

All studies were assessed for risk of bias (ROB) using the Cochrane ROB 2.0 tool in five domains: bias arising from the randomisation process, due to deviations from intended interventions, due to missing outcome data, in the measurement of the outcome and in the selection of the reported result. ${ }^{10}$ The signalling questions were judged as yes, probably yes, probably no, no, or no information, resulting in an overall judgement of study ROB as high, uncertain or low.

\section{Data synthesis and additional analyses}

Study characteristics are presented in a summary of characteristics table. Pooled estimates using the random-effects model, which is a more conservative estimate of treatment effect, were calculated for all treatments combined, then stratified for SGLT2 inhibitors and GLP-1 receptor agonists. All analyses were performed using OpenMeta software. Heterogeneity was quantified by estimating the variance between studies using the $\mathrm{l}^{2}$ statistic.

\section{Results}

After duplications were removed, our initial search identified a possible 761 publications. The initial screen of abstracts reduced this to 103 manuscripts which were reviewed in full (Figure 1). Nine studies met our inclusion criteria, five using GLP-1 receptor agonists and four using SGLT-2 inhibitors. Descriptive details of

Figure 1. PRISMA flow chart

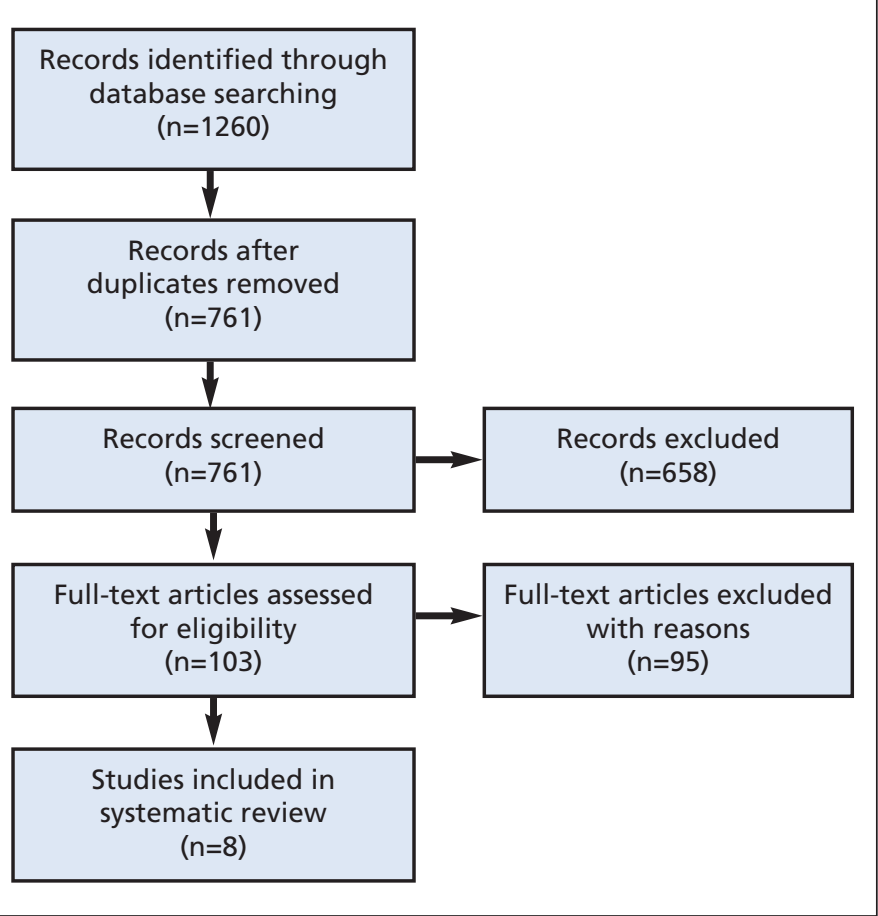


Table 1 Placebo-controlled trials of glucagon-like peptide-1 receptor agonists that reported 3-point MACE outcomes in older adults

\begin{tabular}{|c|c|c|c|c|c|c|c|c|c|}
\hline Trial & Year & $\begin{array}{l}\text { Number } \\
\text { of } \\
\text { participants }\end{array}$ & Drug & $\begin{array}{l}\text { ‘Older' } \\
\text { age }\end{array}$ & $\begin{array}{l}\text { Drug } \\
\text { events }\end{array}$ & $\begin{array}{l}\text { Placebo } \\
\text { events }\end{array}$ & $\begin{array}{l}\text { Hazard } \\
\text { ratio }\end{array}$ & $95 \% \mathrm{Cl}$ & $\begin{array}{l}\% \\
\text { established } \\
\text { CV disease }\end{array}$ \\
\hline SUSTAIN-6 & 2016 & 3297 & Semaglutide & 65 & $55 / 793$ & $76 / 805$ & 0.72 & 0.51 to 1.02 & 83 \\
\hline EXSCEL & 2017 & 14752 & Exenatide & 65 & $426 / 2964$ & $512 / 2975$ & 0.80 & 0.71 to 0.91 & 73 \\
\hline \multirow[t]{2}{*}{ Harmony } & 2018 & 9463 & Albiglutide & $65-74$ & $154 / 1771$ & $166 / 1838$ & 0.97 & 0.78 to 1.21 & 100 \\
\hline & & & & 75 & $50 / 575$ & $69 / 565$ & 0.69 & 0.48 to 1.00 & \\
\hline REWIND & 2019 & 9901 & Dulaglutide & 66 & $331 / 2314$ & $384 / 2350$ & 0.86 & 0.74 to 1.00 & 31 \\
\hline
\end{tabular}

Table 2 Placebo-controlled trials of sodium-glucose co-transporter-2 inhibitors that reported 3-point MACE outcomes in older adults

\begin{tabular}{llllllllll}
\hline Trial & Year & $\begin{array}{l}\text { Number } \\
\text { of } \\
\text { participants }\end{array}$ & Drug & $\begin{array}{l}\text { 'Older' } \\
\text { age }\end{array}$ & $\begin{array}{l}\text { Drug } \\
\text { events }\end{array}$ & $\begin{array}{l}\text { Placebo } \\
\text { events }\end{array}$ & $\begin{array}{l}\text { Hazard } \\
\text { ratio }\end{array}$ & $\begin{array}{l}\mathbf{9 5 \%} \text { Cl } \\
\text { established } \\
\text { CV disease }\end{array}$ \\
\hline Empa-REG & 2015 & 7020 & Empagliflozin & 65 & $239 / 2091$ & $161 / 1036$ & 0.71 & 0.59 to 0.87 & 100 \\
\hline CANVAS* & 2017 & 10142 & Canagliflozin & 65 & $33.8 / 1000$ & $42.3 / 1000$ & 0.80 & 0.67 to 0.95 & 66 \\
DECLARE & 2019 & 17160 & Dapagliflozin & 65 & $390 / 3951$ & $418 / 3956$ & 0.927 & 0.801 to $1.072 \quad 41$ \\
CREDENCE & 2019 & 4401 & Canagliflozin & 65 & $120 / 1009$ & $143 / 1048$ & 0.85 & 0.67 to 1.09 & 50
\end{tabular}

*The CANVAS programme reported events per 1000 patient years therefore, although point estimates can be calculated, these data did not contribute to the meta-analysis.

these studies are presented in Tables 1 and 2. One notable study that could not be incorporated was the ELIXA (Lixisenatide in Patients with Type 2 Diabetes and Acute Coronary Syndrome) trial which did not report any age-specific findings. ${ }^{11}$

\section{Glucagon-like peptide-1 (GLP-1) receptor agonists}

There were five placebo randomised controlled trials of subcutaneously administered GLP-1 receptor agonists, all in addition to standard of care including optimisation of blood pressure and lipid control. Four were post-marketing cardiovascular safety studies that allowed escalation of non-incretin-based therapy in order to match glycaemic control (EXSCEL using exenatide, ${ }^{12}$ LEADER using liraglutide, ${ }^{13}$ HARMONY using albiglutide ${ }^{14}$ and REWIND using dulaglutide ${ }^{15}$ ) with a trial duration ranging from 1.8 to 5.4 years. SUSTAIN-6 testing semaglutide was a placebocontrolled pre-approval regulatory study with a shorter duration and no a priori plan to achieve glycaemic equipoise. ${ }^{16}$ There were differing proportions of those with pre-existing cardiovascular disease compared with those at high cardiovascular risk in these trials; however, all studies were well balanced through randomisation (Table 1). Of these studies, EXSCEL ${ }^{16}$ achieved the primary endpoint of non-inferiority compared with placebo whereas those using GLP-1 analogues (ie, liraglutide, albiglutide, dulaglutide and semaglutide) demonstrated superiority compared with placebo in reducing cardiovascular events. These studies used a nominal cut-off for 'elderly' of $\geq 60, \geq 65$ and/or $\geq 75$ years. In the case of EXSCEL and HARMONY, there were dual cut-offs with $65-75$ and $\geq 75$ years being reported separately. In total there were 25,219 older adults in these studies, with 1,604 events in those on treatment compared with 1,880 in the placebo group (OR 0.847 (95\% Cl 0.788 to 0.910$)$, test for heterogeneity $\mathrm{I}^{2}=0 \%, \mathrm{p}=0.7$; Figure $\left.2 \mathrm{~A}\right)$. The absolute risk reduction was $1.94 \%$ with a number needed to treat of 51.5 over 3.3 years to prevent a MACE. There was a numerically similar $16 \%$ benefit in the younger participants in the studies (OR $0.840(95 \% \mathrm{Cl}$ 0.698 to 1.011 ); Figure $3 A$ ), although this was not statistically significant despite exploring outcomes in a similar number of patients $(n=22,824)$. There was, however, significant heterogeneity in the results of the younger adults, $\left(I^{2}=73 \%, p=0.005\right)$, such that the EXSCEL study was to the right of the line of unity demonstrating a point estimate in favour of placebo with confidence intervals crossing 1 . The absolute risk reduction among the agents in younger adults was $0.94 \%$, requiring a number needed to treat of 106 participants to prevent one event.

\section{Sodium-glucose co-transporter-2 (SGLT-2) inhibitors}

Four studies met our a priori selection criteria; however, one study (The Canagliflozin and Cardiovascular and Renal Events in Type 2 Diabetes; The CANVAS program) reported their findings in events per 1,000 patient years rather than absolute numbers, 
rendering it impossible to include these data in the meta-analysis. ${ }^{17}$ The findings from CANVAS are therefore presented in the summary in Table 2, but not in the meta-analysis (Figure 2B).

The remaining three studies explored the role of empagliflozin after an acute coronary event (EMPA-REG), ${ }^{18}$ dapagliflozin in those with established or at high risk of developing cardiovascular disease (DECLARE) ${ }^{19}$ and canagliflozin in renal im- pairment (estimated glomerular filtration rate (eGFR) 30-90 $\mathrm{mL} / \mathrm{min}$; CREDENCE). ${ }^{20}$ All studies were placebo controlled in addition to standard of care with the intention of achieving glycaemic equipoise and met their primary endpoint of cardiovascular safety. EMPA-REG and CREDENCE achieved superiority compared with placebo in the total population whereas, in DECLARE, dapagliflozin only achieved the latter of the two

Figure 2. Major adverse cardiovascular events (MACE) in placebo-controlled trials comparing (A) glucagon-like peptide-1 receptor agonists (GLP-1RA) and (B) sodium-glucose co-transporter-2 inhibitors (SGLT-2i) in addition to standard of care in older adults ( $>65$ years)

(A)

Studies

LEADER $>65$ year old 2016 SUSTAIN-6 $>65$ year old 2016 EXSCEL $>65$ year old 2017 HARMONEY >65 year old 2018 REWIND > 65 year old 2019

Overall $\left(\left.\right|^{\wedge} 2=0 \%, p=0.746\right)$

(B)

\section{Studies}

Empa-Reg $>65$ year old 2015 DECLARE >65 year old 2019 CREDENCE >65 year old 2019

Overall $\left(\left.\right|^{\wedge} 2=5478 \%, p=0.110\right)$

$\begin{array}{lcc}\text { Estimate }(95 \% \mathrm{Cl}) & \text { MACE/GLP-1RA } & \text { MACE/Placebo } \\ 0.891(0.779,1.019) & 468 / 3471 & 528 / 3548 \\ 0.715(0.498,1.026) & 55 / 793 & 76 / 805 \\ 0.812(0.717,0.920) & 546 / 3573 & 657 / 3616 \\ 0.875(0.718,1.065) & 204 / 2356 & 235 / 2403 \\ 0.855(0.728,1.003) & 331 / 2314 & 384 / 2350 \\ 0.847(0.788,0.910) & 1604 / 12507 & \mathbf{1 8 8 0 / 1 2 7 2 2}\end{array}$

\section{4}

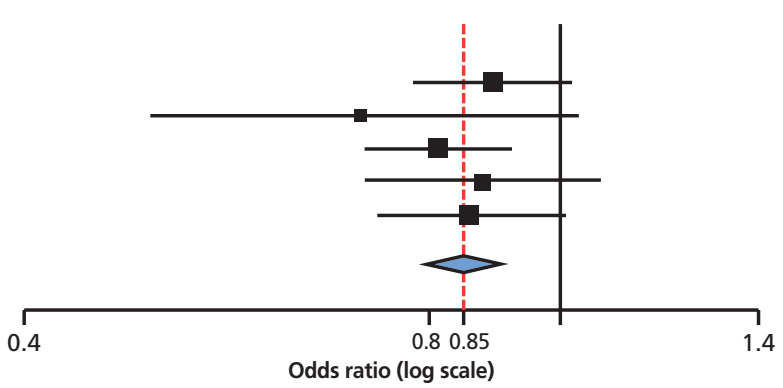

Odds ratio (log scale)

$\begin{array}{lcc}\text { Estimate }(95 \% \mathrm{Cl}) & \text { MACE/SGLT-2i } & \text { MACE/Placebo } \\ 0.701(0.565,0.870) & 239 / 2091 & 161 / 1036 \\ 0.927(0.801,1.072) & 390 / 3951 & 418 / 3956 \\ 0.854(0.659,1.108) & 120 / 1009 & 143 / 1048 \\ \mathbf{0 . 8 3 1}(\mathbf{0 . 6 9 9 , 0 . 9 8 9 )} & \mathbf{7 4 9 / 7 0 5 1} & \mathbf{7 2 2 / 6 0 4 0}\end{array}$

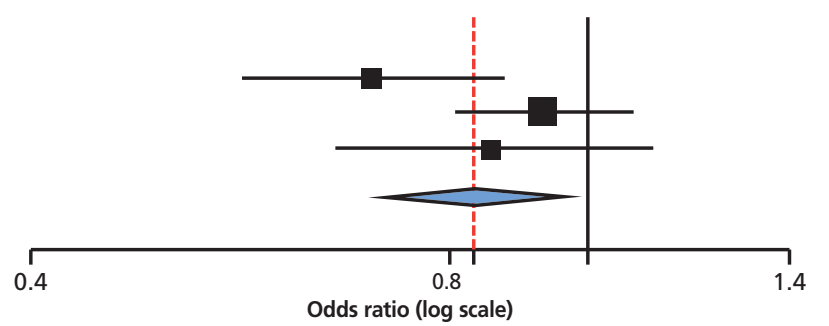

Figure 3. Major adverse cardiovascular events (MACE) in placebo-controlled trials comparing (A) glucagon-like peptide-1 receptor agonists (GLP-1RA) and (B) sodium-glucose co-transporter-2 inhibitors (SGLT-2i) in addition to standard of care in older adults ( $>65$ years).

(A)

Studies

LEADER 2016

SUSTAIN-6 2016

EXSCEL 2017

HARMONEY 2018

REWIND 2019

Overall $\left(I^{\wedge} 2=7296 \%, p=0.005\right)$

(B)

Studies

Empa-Reg 2015

DECLARE 2019

CREDENCE 2019

Overall $\left(\left.\right|^{\wedge} 2=4954 \%, p=0.138\right)$
Estimate $(95 \% \mathrm{Cl})$

$0.764(0.601,0.973)$

$0.768(0.531,1.112)$

$1.064(0.920,1.230)$

$0.659(0.524,0.828)$

$0.923(0.773,1.103)$

$0.840(0.698,1.011)$

$\begin{array}{cc}\text { MACE/GLP-1RA } & \text { MACE/Placebo } \\ 140 / 1197 & 166 / 1124 \\ 53 / 855 & 70 / 884 \\ 413 / 4392 & 393 / 4421 \\ 134 / 2385 & 193 / 2329 \\ 263 / 2635 & 279 / 2602 \\ 1003 / 11464 & 1101 / 11360\end{array}$

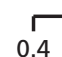

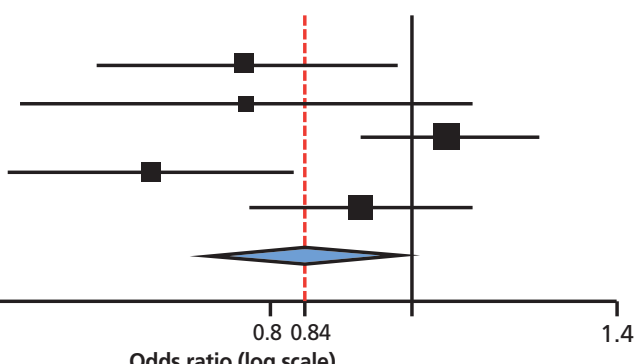

Odds ratio (log scale)

$\begin{array}{cc}\text { MACE/SGLT-2i } & \text { MACE/Placebo } \\ 251 / 2596 & 121 / 1297 \\ 366 / 4631 & 385 / 4822 \\ 97 / 1193 & 123 / 1151 \\ \mathbf{7 1 4 / 8 4 2 0} & \mathbf{6 2 9 / 7 2 7 0}\end{array}$

$714 / 8420$
Estimate $(95 \% \mathrm{Cl})$

$1.040(0.828,1.307)$

$0.989(0.852,1.148)$

$0.740(0.559,0.978)$

$0.936(0.787,1.113)$

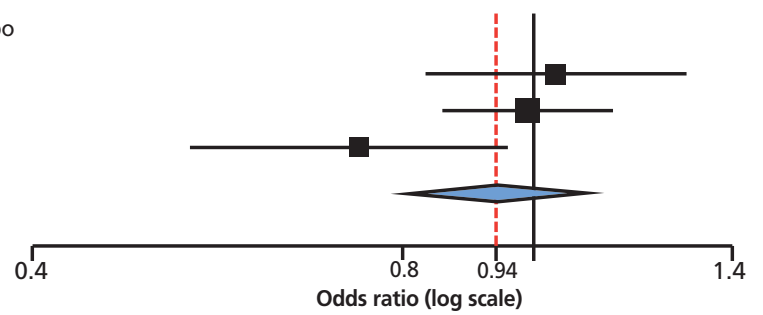


endpoints from the split alpha between 3-point MACE and hospitalisations due to heart failure.

In combination, there were 13,091 older adults considered in the analysis of the SGLT-2 inhibitors (Figure 2B). The 749 events in the 7,051 individuals in the treatment group (10.62\%) was superior to the 722 events in the 6,040 individuals in the placebo group (11.95\%) by $17 \%$ (OR 0.831 (95\% Cl 0.699 to $0.989)$ : test for heterogeneity $\left.l^{2}=54.8 \%, p=0.11\right)$. This absolute risk reduction of $1.33 \%$ translates to a number needed to treat of 75 people for 3.5 years to prevent one cardiovascular event. This compares favourably to younger patients in whom there was a non-significant $0.2 \%$ absolute risk reduction (OR 0.936 (95\% Cl 0.787 to 1.113); Figure 3B). Of interest, the point estimates of CANVAS program for both older (OR $0.80(95 \% \mathrm{Cl}$ 0.67 to 0.95$)$ ) and younger (OR 0.91 ( $95 \% \mathrm{Cl} 0.76$ to 1.10$)$ ) participants was similar to the calculated odds ratio in the other studies, suggesting that, had they reported absolute numbers and been included in the meta-analysis, they would not have materially altered the findings.

\section{Discussion}

This systematic review has demonstrated the benefit of treatment with a GLP-1 receptor agonist or an SGLT-2 inhibitor in older adults who were eligible for inclusion in these large randomised controlled trials. In both cases the agents of interest were beneficial in older adults, with a smaller number of patients required to be treated in order to gain benefit. Indeed, in the case of SGLT-2 inhibitors, benefit was only seen in older adults whereas in younger populations no significant benefit was demonstrated. This is of particular relevance given the recent update to the UK guidance for the management of older adults with diabetes, which emphasises the need to assess frailty in individuals and subsequently individualise the treatment options. ${ }^{3}$ This stakeholder's document highlighted the observation that weight loss in older adults has no proven benefit; indeed, it may exacerbate sarcopenia and thus have a detrimental impact on prognosis. ${ }^{21}$ As a result, the stakeholder's position statement recommended caution in the use of agents that may promote weight loss. The current analysis suggests, however, that fitter older adults, who would have been eligible for inclusion in clinical trials, would benefit from treatment with SGLT-2 inhibitors or GLP-1 analogues.

It is important to acknowledge, however, that older clinical trial participants may not be truly representative of the general population. Indeed, the term 'older adults' can span more than 35 years, from 65 years to centenarians, with some being robust in employment and independently active whilst others are in the terminal decline of multi-morbidity and frailty. The physiological differences attributable to frailty are substantial; however, current treatment strategies are based on extrapolation from the outcomes in chronologically matched patients. ${ }^{22}$ Frail older adults are rarely included in clinical trials, with exclusion criteria to recruitment such as polypharmacy, cognitive decline and multiple co-morbidities being common. ${ }^{23}$ For this reason, older adults included in cardiovascular outcome trials tend to be

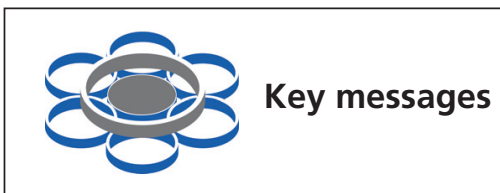

- Unintentional weight loss is a poor prognostic indicator in older adults with type 2 diabetes

- There has been concern regarding the use of sodium-glucose co-transporter-2 (SGLT-2) inhibitors and glucagon-like peptide-1 (GLP-1) receptor agonists in older adults because of their impact on weight

- This systematic review and meta-analysis showed that major adverse cardiovascular events outcomes were reduced in older adults with both agents: in the case of GLP-1 receptor agonists, to a similar degree as seen in younger adults; in the case of SGLT-2 inhibitors, numerically superior to the benefit in younger populations

- Whereas reassuring for robust older adults, this should not be extrapolated to frail patients without further research

biologically healthier than the general older population. ${ }^{24}$ Even within these populations, trials of glycaemic control in older adults highlight an exaggerated 'U-shaped' curve with higher mortality at both low and higher $\mathrm{HbA}_{1 \mathrm{c} .}{ }^{25,26}$ To date there are very limited data on the optimal medication choices for frail older adults, and fewer still on the individualisation of care in those living with frailty. ${ }^{27}$ There is, however, a pressing need to explore such populations, given the significant impact that heart failure and stroke have on functional capacity in older adults. These complications of diabetes are significant contributors to frailty progression. The co-existence of heart failure and diabetes has a poorer prognosis than many cancers. ${ }^{28}$ An anticipated life expectancy of less than 7 years is a common exclusion criterion, ${ }^{29}$ which would thereby exclude the patients with the most to gain in our analysis from the studies to evaluate their efficacy.

This systematic review has highlighted the importance and potential benefits of using both GLP-1 agonists and SGLT-2 inhibitors to reduce cardiovascular outcomes in older adults with type 2 diabetes. A key limitation is that only eight studies were eligible for inclusion. Therefore, it is very important that further research in this field is undertaken. It is also important to acknowledge that 3-point MACE does not represent the only outcome of interest for older adults living with diabetes. It is accepted that SGLT-2 inhibitors, as a class, improve outcomes for people living with heart failure. Hospitalisation from heart failure is important for older adults with diabetes; however, these outcomes are not stratified by age and thus were not included in this meta-analysis. In a population in whom weight loss is associated with frailty and poor cardiovascular outcomes, the absence of any adverse cardiovascular signals should be reassuring to use SGLT-2 inhibitors in older adults with heart failure. 


\section{Conclusions}

On the basis of these data, we would advocate the use of GLP1 analogues and SGLT-2 inhibitors in any older adult with mild or moderate frailty, particularly when the frailty is a function of cardiovascular disease itself. Further research is required to determine whether the benefits are extended to older adults with severe frailty or patients at lower risk of cardiovascular events.

Acknowledgements WDS would like to acknowledge the support of the NIHR Exeter Clinical Research Facility and the NIHR Collaboration for Leadership in Applied Health Research and Care (CLAHRC) for the South West Peninsula.

Conflict of interest WDS holds research grants from Novo Nordisk and Takeda and has received speaker fees from AstraZeneca, Bayer, Napp, NovoNordisk and Takeda. JG declares no conflicts of interest. WDS would like to add that the views expressed in this publication are those of the authors and not necessarily those of the NIHR Exeter Clinical Research Facility, the NHS, the NIHR or the Department of Health in England.

Authorship Both authors meet the International Committee of Medical Journal Editors (ICMJE) criteria for authorship for this article, take responsibility for the integrity of the work as a whole, and have given their approval for this version to be published.

Compliance with ethics guidelines This was a retrospective meta-analysis of published data. All studies incorporated in the analysis had been through appropriate ethics review and approvals

Funding None.

\section{References}

1. Forouhi NG, Wareham NJ. Epidemiology of diabetes. Medicine (Abingdon) 2014:42(12):698-702. https://doi.org/10.1016/.mpmed.2014.09.007

2. Sinclair AJ, Gadsby R, Penfold S, Croxson SC, Bayer AJ. Prevalence of diabetes in care home residents. Diabetes Care 2001;24:1066-8. https://doi.org/10.2337/diacare.24.6.1066

3. Strain WD, Hope SV, Green A, Kar P, Valabhji J, Sinclair AJ. Type 2 diabetes mellitus in older people: a brief statement of key principles of modern day management including the assessment of frailty. A national collaborative stakeholder initiative. Diabet Med 2018;35(7):838-45. https://doi.org/ 10.1111/dme.13644

4. Grieve DJ, Cassidy RS, Green BD. Emerging cardiovascular actions of the incretin hormone glucagon-like peptide-1: potential therapeutic benefits beyond glycaemic control? Br J Pharmacol 2009;157(8):1340-51. https://doi.org/10.1111/j.1476-5381.2009.00376.x

5. Mehta A, Marso SP, Neeland IJ. Liraglutide for weight management: a critical review of the evidence. Obes Sci Pract 2017:3(1):3-14. https://doi.org/10.1002/osp4.84

6. Chao EC, Henry RR. SGLT2 inhibition--a novel strategy for diabetes treatment. Nat Rev Drug Discov 2010;9(7):551-9. https://doi.org/ $10.1038 /$ nrd3180

7. Garg V, Verma S, Connelly K. Mechanistic insights regarding the role of SGLT2 inhibitors and GLP1 agonist drugs on cardiovascular disease in diabetes. Prog Cardiovasc Dis 2019;62(4):349-57. https://doi.org/10.1016/ j.pcad.2019.07.005

8. Shamseer L, Moher D, Clarke M, et al. Preferred Reporting Items for Systematic Review and Meta-Analysis Protocols (PRISMA-P) 2015: elaboration and explanation. BMJ 2015;350:g7647. https://doi.org/10.1136/bmj.g7647. Correction. BMJ 2016;354:i4086. https://doi.org/10.1136/bmj.i4086

9. Strain WD, Griffiths J. The impact of SGLT-2 inhibitors and GLP-1 analogues on cardiovascular outcomes in non-frail older adults. PROSPERO 2020; CRD42020200601. Available from: https://www.crd.york.ac.uk/prospero/display_record.php?|D=CRD42020200601

10. Moseley AM, Rahman P, Wells GA, et al. Agreement between the Cochrane risk of bias tool and Physiotherapy Evidence Database (PEDro) scale: a metaepidemiological study of randomized controlled trials of physical therapy interventions. PLoS One 2019;14(9):e0222770. https://doi.org/10.1371/ journal.pone. 0222770
11. Pfeffer MA, Claggett B, Diaz R, et al. Lixisenatide in patients with type 2 diabetes and acute coronary syndrome. N Eng/ J Med 2015;373(23):224757. https://doi.org/10.1056/NEJMoa1509225

12. Holman RR, Bethel MA, Mentz RJ, et al. Effects of once-weekly exenatide on cardiovascular outcomes in type 2 diabetes. N Engl J Med 2017; 377(13):1228-39. https://doi.org/10.1056/NEJMoa1612917

13. Marso SP, Daniels GH, Brown-Frandsen K, et al. Liraglutide and cardiovascular outcomes in type 2 diabetes. N Engl J Med 2016;375(4):311-22. https://doi.org/10.1056/NEJMoa1603827

14. Hernandez AF, Green JB, Janmohamed S, et al. Albiglutide and cardiovascular outcomes in patients with type 2 diabetes and cardiovascular disease (Harmony Outcomes): a double-blind, randomised placebo-controlled trial. Lancet 2018;392(10157):1519-29. https://doi.org/10.1016/S01406736(18)32261-X

15. Gerstein HC, Colhoun HM, Dagenais GR, et al. Dulaglutide and cardiovascular outcomes in type 2 diabetes (REWIND): a double-blind, randomised placebo-controlled trial. Lancet 2019;394(10193):121-30. https://doi.org/ 10.1016/S0140-6736(19)31149-3

16. Marso SP, Bain SC, Consoli A, et al. Semaglutide and cardiovascular outcomes in patients with type 2 diabetes. N Eng/ J Med 2016;375(19):183444. https://doi.org/10.1056/NEJMoa1607141

17. Neal B, Perkovic V, Mahaffey KW, et al. Canagliflozin and cardiovascular and renal events in type 2 diabetes. N Engl J Med 2017;377(7):644-57. https://doi.org/10.1056/NEJMoa1611925

18. Zinman B, Wanner C, Lachin JM, et al. Empagliflozin, cardiovascular outcomes, and mortality in type 2 diabetes. N Engl J Med 2015;373(22):211728. https://doi.org/10.1056/NEJMoa1504720

19. Wiviott SD, Raz I, Bonaca MP, et al. Dapagliflozin and cardiovascular outcomes in type 2 diabetes. N Engl J Med 2019;380(4):347-57. https://doi.org/10.1056/NEJMoa1812389

20. Mahaffey KW, Jardine MJ, Bompoint S, et al. Canagliflozin and cardiovascular and renal outcomes in type 2 diabetes mellitus and chronic kidney disease in primary and secondary cardiovascular prevention groups. Circulation 2019;140(9):739-50. https://doi.org/10.1161/CIRCULATIONAHA.119.042007

21. Kamel HK, labal MA. Body mass index and mortality among hospitalized elderly patients. Arch Intern Med 2001;161(11):1459-60. https://doi.org/ 10.1001/archinte.161.11.1459

22. Lang PO, Michel JP, Zekry D. Frailty syndrome: a transitional state in a dynamic process. Gerontology 2009;55(5):539-49. https://doi.org/ $10.1159 / 000211949$

23. Cruz-Jentoft AJ, Carpena-Ruiz M, Montero-Errasquin B, Sanchez-Castellano C, Sanchez-Garcia E. Exclusion of older adults from ongoing clinical trials about type 2 diabetes mellitus. J Am Geriatr Soc 2013;61(5):734-8. https://doi.org/10.1111/jgs.12215

24. Sinclair AJ, Abdelhafiz AH, Forbes A, Munshi M. Evidence-based diabetes care for older people with type 2 diabetes: a critical review. Diabet Med 2019;36(4):399-413. https://doi.org/10.1111/dme.13859

25. Bruce DG, Davis WA, Davis TME. Glycaemic control and mortality in older people with type 2 diabetes: The Fremantle Diabetes Study Phase II. Diabetes Obes Metab 2018;20(12):2852-9. https://doi.org/10.1111/dom.13469

26. Forbes A, Murrells T, Mulnier H, Sinclair AJ. Mean HbA1c, HbA1c variability, and mortality in people with diabetes aged 70 years and older: a retrospective cohort study. Lancet Diabetes Endocrinol 2018;6(6):476-86. https://doi.org/10.1016/S2213-8587(18)30048-2

27. Strain WD, Lukashevich V, Kothny W, Hoellinger MJ, Paldanius PM. Individualised treatment targets for elderly patients with type 2 diabetes using vildagliptin add-on or lone therapy (INTERVAL): a 24 week, randomised, double-blind, placebo-controlled study. Lancet 2013;382(9890):409-16. https://doi.org/10.1016/S0140-6736(13)60995-2

28. MacDonald MR, Petrie MC, Varyani F, et al. Impact of diabetes on outcomes in patients with low and preserved ejection fraction heart failure: an analysis of the Candesartan in Heart failure: Assessment of Reduction in Mortality and morbidity (CHARM) programme. Eur Heart J 2008;29(11):1377-85. https://doi.org/10.1093/eurheartj/ehn153

29. Duckworth W, Abraira C, Moritz T, et al. Glucose control and vascular complications in veterans with type 2 diabetes. N Engl J Med 2009;360(2):12939. https://doi.org/10.1056/NEJMoa0808431 